\title{
AFFINE GEOMETRY: A LATTICE CHARACTERIZATION
}

\author{
M. K. BENNETT
}

\begin{abstract}
Necessary and sufficient conditions are given for a lattice $L$ to be the
\end{abstract} lattice of flats of an affine space of arbitrary (possibly infinite) dimension.

1. Incidence spaces and Hilbert lattices. By an incidence space [G1], we mean a system of points, lines, and planes satisfying Hilbert's Axioms of Incidence [Verknüpfung] [H1] as follows:

(I1) Any two distinct points determine a unique line.

(I2) Any three noncollinear points determine a unique plane.

(I3) If two planes in a three-space intersect, their intersection is a line ((I3) has been modified, as Hilbert was considering only three-dimensional geometry).

(I4) If two points are in a plane, the line they determine is in that plane.

(I5) There are at least two points on each line.

The flats (sets of points which contain, together with any two points, the line they determine) of an incidence space form a complete lattice under set theoretical inclusion; such lattices were characterized by Sasaki and Wyler in the fifties. (In case each line contains exactly two points, one must also postulate that a flat contains, together with any three noncollinear points, the plane they determine.)

THEOREM (SASAKI [S1], WYLER [W1]). $L$ is the lattice of flats of an incidence space if and only if

(1) $L$ is compactly atomistic,

(2) $M(a, b)$ implies $M(b, a)$ for all $a, b$ in $L$,

(3) $a \wedge b \neq 0$ implies $M(a, b)$ in $L$.

We use compactly atomistic to mean $L$ is complete; each nonzero element of $L$ is the join of atoms (minimal nonzero elements, or points); and if an atom is under a join of atoms, it is under a finite subjoin of those atoms. The expression $M(a, b)$ means that $a$ and $b$ form a modular pair. (For the definitions of standard lattice-theoretic terms, see, for example, [B1].) Lattices satisfying (1) and (2) are sometimes called geometric lattices [M1]; in [B1] the term geometric lattice is used for atomic lattices of finite height satisfying (2). We shall use the term Hilbert lattice for a lattice satisfying (1), (2) and (3).

Received by the editors February 22, 1982 and, in revised form, September 24, 1982.

AMS (MOS) subject classifications (1970). Primary 06A25, 50D05. 
Two lines in an incidence space are said to be parallel if they are coplanar and nonintersecting; and an incidence space is called an affine space (see [A1, S3]) if it satisfies the parallel axiom, given in two parts below;

(P1) (Uniqueness) Through any point not on a line, there is at most one parallel to the line,

(P2) (Existence) Through any point not on a line, there is at least one parallel to the line.

Sasaki essentially translated the parallel axiom into lattice-theoretical terms in order to obtain his characterization of the lattice of flats of an affine space.

THEOREM (SASAKI [S2]). $L$ is the lattice of flats of an affine space if and only if $L$ is a Hilbert lattice satisfying:

(4) For $p, q$ and $r$ atoms of $L$ such that $p \wedge(q \vee r)=0$, there is a unique $m$ in $L$ such that $p<m<p \vee q \vee r$, and $m \wedge(q \vee r)=0$.

Our aim is to give a characterization of the lattice of flats of an affine space in terms more natural to a lattice-theoretic setting. Synthetic axioms for geometry distinguish 'lines' and 'planes' from 'points'; whereas the essential novelty of the lattice theoretic approach to a geometry is that it treats all flats as being equally basic. This is the spirit of the characterizations by Birkhoff [B2] and Menger [M2] of projective geometries in terms of irreducible complemented modular lattices. (See the remarks of G. Birkhoff on this subject in Ordered sets (I. Rival, ed.), Reidel, Dordrecht, 1981.)

2. Wilcox lattices and $K_{6}$. A projective space is an incidence space such that there are at least three points on each line, and any two coplanar lines intersect. As is well known, the concepts of projective and affine space are coextensive; in particular, one can obtain an affine space by deleting a hyperplane (maximal proper flat) from a projective space, and this construction reverses. In lattice-theoretic terms, this means that any affine geometry is a subset of a projective geometry, obtained by removing a coatom (maximal element different from 1 ) and all nonzero elements beneath it. (Here we shall use 'geometry' to refer to a lattice of flats, as opposed to a synthetic 'space'.) We shall define a more general class of lattices, first investigated by L. R. Wilcox in the thirties (see [M1]).

Let $(M, \sqcup, \Pi)$ be a modular lattice. Let $S$ be a subset of $M$ such that 0 is not in $S$, but $S \cup 0$ is a proper ideal (i.e., for $a$ and $b$ in $S, a \bigsqcup b$ is in $S$; and for $a$ in $S$ with $0 \neq c<a, c$ is in $S)$. Then $(L, \vee, \wedge)$ is a lattice where

(i) $a \vee b=a \bigsqcup b$,

(ii) $a \wedge b=\left\{\begin{array}{l}0 \text { if } a \sqcap \mid b \in S \\ a \sqcap \mid b \text { otherwise }\end{array}\right.$.

We shall call such a lattice $L$ a Wilcox lattice, and we note that any affine geometry is a Wilcox lattice where $M$ is a projective geometry and $S$ is the set of nonzero elements of the interval sublattice $[0, h]$ for $h$ a coatom of $M$.

In an incidence geometry, if intersecting lines $m$ and $m^{\prime}$ are both parallel to line $l$, the sublattice generated by $l, m$ and $m^{\prime}$ is the six-element lattice shown in Figure 1 . 


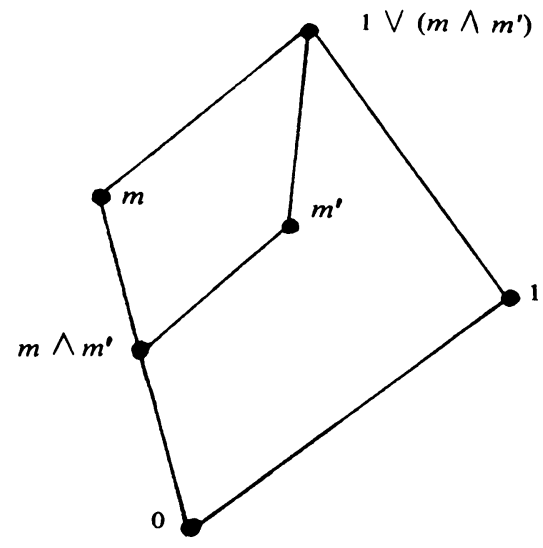

FIGURE 1

It turns out that no lattice isomorphic to the one in Figure 1 may appear anywhere as a sublattice of a Wilcox lattice (regardless of the heights of the elements involved).

2.1. TheOREM. Let $L$ be a Wilcox lattice. Then $L$ does not contain $K_{6}$ as a sublattice, where $K_{6}$ is the lattice shown in Figure 2.

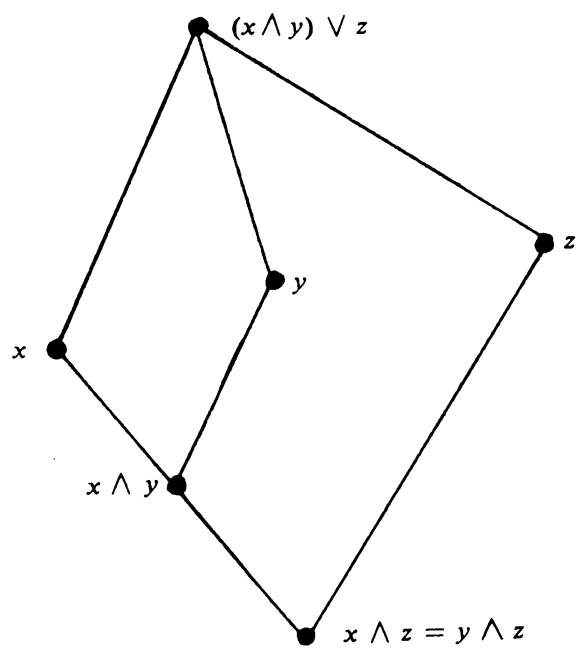

FIGURE 2

Proof. We shall use the notation $(M, \sqcup, \sqcap),(L, \vee, \wedge)$ and $S$ as above. We first note that if $a \wedge b \neq 0$ in $L$, then $M(a, b)$ holds; hence if $L$ contains $K_{6}$ as a sublattice, its bottom element must be 0 . Also, $x \sqcap z \neq 0$ (in $M$ ); otherwise $((x \sqcap y) \sqcup z) \sqcap x=x$, while $(x \sqcap y) \sqcup(z \sqcap x)=x \sqcap y$, a contradiction of the modularity of $M$. Similarly, $y \sqcap z \neq 0$; hence $x \sqcap z$ and $y \sqcap z$ are in $S$.

Since

$$
\begin{aligned}
x \sqcap(y \sqcup(x \sqcap z)) & =(x \sqcap y) \bigsqcup(x \sqcap z)=((x \sqcap y) \sqcup z) \sqcap x \\
& =(x \bigsqcup z) \sqcap x=x,
\end{aligned}
$$


we have $x \leqslant y \bigsqcup(x \sqcap z)$, and since $y \leqslant y \bigsqcup(x \sqcap z)$, it follows that $z \leqslant y \bigsqcup(x \sqcap z)$. Thus

$$
z=z \sqcap(y \bigsqcup(x \sqcap z))=(z \sqcap y) \bigsqcup(x \sqcap z) \in S,
$$

so $z$ is not in $L$, and $L$ does not contain $K_{6}$ as a sublattice.

We can combine this result with Theorem 20.10 in [M1] to obtain

2.2. Corollary. Let $L$ be a Hilbert lattice of height $\geqslant 4$. Then $L$ is a Wilcox lattice if and only if $L$ does not contain $K_{6}$ as a sublattice.

3. The characterization theorem. We say two elements $x$ and $y$ in a Hilbert lattice are parallel (written $x / / y$ ) if $x \wedge y=0$ and $x \vee y$ covers $x$ and $y$ (where $a$ covers $b$, $a>b$ means that $a$ is greater than $b$ and there is no element between them). This definition implies that parallel elements have the same dimension; and in case $x$ and $y$ are coatoms, they are parallel if and only if they are complementary. In case $x$ and $y$ are lines (elements of height 2) this definition of parallelism agrees with the definition of parallel lines given in $\$ 1$.

The connection between modularity and parallelism of lines in a Hilbert lattice is shown in the lemma below.

3.1. Lemma. Let $a$ and $b$ be elements of $a$ Hilbert lattice L. Then $M(a, b)$ fails if and only if $a \wedge b=0$ and there are lines $1 \leqslant a, m \leqslant b$ with $1 / / m$.

Proof. Suppose first that $M(a, b)$ fails. Then there exists $y \leqslant b$ such that $y \vee(a \wedge b)=y \neq(y \vee a) \wedge b$. If, for each atom $s \leqslant b,(s \vee a) \wedge b=s$, we arrive at a contradiction as follows. There is an atom $r \leqslant(y \vee a) \wedge b$ with $r \$ y$. Since $r \leqslant y \vee a, r \leqslant y_{1} \vee \cdots \vee y_{n} \vee a$, where the $y_{1}$ are atoms under $y$. But then

$$
\begin{aligned}
r & \leqslant\left(\left(y_{1} \vee \cdots \vee y_{n-1}\right) \vee\left(y_{n} \vee a\right)\right) \wedge b \\
& =\left(y_{1} \vee \cdots \vee y_{n-1}\right) \vee\left(\left(y_{n} \vee a\right) \wedge b\right) \\
& =y_{1} \vee \cdots \vee y_{n-1} \vee y_{n} \leqslant y,
\end{aligned}
$$

a contradiction.

Thus for some atom $s \leqslant b, s \neq(s \vee a) \wedge b$, whence there is an atom $t \neq s$ with $t \leqslant(s \vee a) \wedge b$. Since $(s \vee a) \wedge(s \vee t)=s \vee t$, and $s \vee(a \wedge(s \vee t))=s$. $M(a, s \vee t)$ fails. But this implies that $M(s \vee t, a)$ fails, and by the argument used above, there are atoms $s^{\prime}$ and $t^{\prime}$ under $a$ such that $M\left(s \vee t, s^{\prime} \vee t^{\prime}\right)$ fails. Let $l=s^{\prime} \vee t^{\prime}$ and $m=s \vee t$. Since $M(l, m)$ fails, $l \wedge m=0$. For some atom $p \leqslant m$, we must have $p<(p \vee l) \wedge m$; hence $(p \vee l) \wedge m=m$, which implies that $p \vee l=l$ $\vee m$. Thus $l \vee m$ is of height 3 , and covers both $l$ and $m$, therefore $l / / m$.

Conversely, suppose that $a \wedge b=0$ with $l \leqslant a, m \leqslant b$, and $l / / m$. Then for $p$ an atom under $m$, we have $(p \vee l) \wedge m=m$; hence $(p \vee a) \wedge b \geqslant m>p$ and $M(a, b)$ fails.

One may use standard lattice-theoretic arguments to show that each element (except 1) in a Hilbert lattice is under some coatom, and then modify the proof of 18.9 in [M1] to obtain a condition on Hilbert lattices which implies the existence part of the parallel axiom (P2). 
3.2. Lemma. Let $L$ be a Hilbert lattice such that each coatom of $L$ has a complementary coatom. Then for any line $l$ in $L$, there exists a line $m$ in $L$ such that $l / / m$.

Proof. Let $h$ be a coatom such that $l \leqslant h$, and let $k$ be a complementary coatom for $h$. Then $M(k, l)$ fails since for $q$ an atom under $l,(q \vee k) \wedge l=l \neq q$. Thus there is a line $m$ under $k$ such that $M(l, m)$ fails, hence $l / / m$.

3.3. Lemma. Let $L$ be a Hilbert lattice of height $\geqslant 4$ such that each coatom has a complementary coatom. Then the existence part of the parallel axiom holds in L.

Proof. Let $r$ be an atom of $L$, and $l$ a line such that $r \wedge l=0$. By 3.2 there is a line $m$ such that $l / / m$. If $r \leqslant m$, we are done. Suppose $r \neq l \vee m$. Then $r \vee l \vee m$ covers $l \vee m$, whence $(r \vee l) \wedge(r \vee m)$ is of height 2, a line. But $((r \vee l) \wedge(r \vee$ $m) \wedge l=(r \vee m) \wedge l$, and if $t$ were an atom under $(r \vee m) \wedge l$, then $r \leqslant(t \vee m)$ $\leqslant l \vee m$, a contradiction. Thus $((r \vee l) \wedge(r \vee m)) \wedge l=0$, and

$$
((r \vee l) \wedge(r \vee m)) \vee l=(r \vee l) \wedge(r \vee m \vee l)=r \vee l
$$

which covers $l$. Thus $(r \vee l) \wedge(r \vee m) / / l$.

If $r \leqslant l \vee m$, take $r^{\prime} \$ l \vee m$ (which is where we need $L$ of height $\geqslant 4$ ) and get $r^{\prime} \leqslant m^{\prime} / / l$. Then $r^{\prime} \leqslant m^{\prime} \vee l$, and if $r \leqslant m^{\prime} \vee l$, we would have $r \leqslant r^{\prime} \vee l$, which implies $r^{\prime} \leqslant r \vee l \leqslant m \vee l$, a contradiction. Thus $r \$ m^{\prime} \vee l$, and we can use the argument above to show that there is a line $m_{1}$ such that $r \leqslant m_{1}$ and $m_{1} / / l$.

We can now prove the characterization theorem.

3.4. THEOREM. Let $L$ be a Hilbert lattice of height different from 3 (or a finite Hilbert lattice of height 3 ). Then $L$ is an affine geometry if and only if

(1) L does not contain $K_{6}$ as a sublattice, and

(2) each coatom of $L$ has a complementary coatom.

Proof. If $L$ is an affine geometry (of any dimension) then $L$ is a Hilbert lattice by the Sasaki-Wyler theorem; (1) holds by Theorem 2.1 above; and (2) is implied by the stronger 'parallel axiom for hyperplanes', through a point not on a hyperplane there is a unique parallel hyperplane.

Conversely, if $L$ is of height 1 or 2 , it is an affine geometry in a trivial sense. If the height of $L$ is 4 or greater, then $\mathrm{L}$ is an incidence geometry by the Sasaki-Wyler theorem; the uniqueness part of the parallel axiom holds since $L$ cannot have the lattice of Figure 1 as a sublattice; and the existence part of the parallel axiom holds by 3.3 .

If $L$ is finite of height 3 , the existence part of the parallel axiom may be proved by a counting argument. One first shows that any two intersecting lines have the same number of points. Furthermore, by 3.2, each line has a parallel. If $p$ is a point not in line $l$, and there is no line through $p$ parallel to $l$, then the number of points on $l$ is equal to the number of lines through $p$. If $m$ is some line through $p$, and $m^{\prime}$ is parallel to $m$, then $m^{\prime} \wedge l \neq 0$. Then $\# l=\# m^{\prime}$. But the number of points on $m^{\prime}$ is one less than the number of lines through $p$, a contradiction.

The author is indebted to the referee for providing an example of an infinite lattice of height 3 which satisfies the hypotheses of 3.4, but which is not an affine 
geometry. This example consists of the flats of the incidence structure $G_{\infty}$, described below.

Given a finite set of points and lines, $G_{i}$, not satisfying (I1), order the points by labelling then $1, \ldots, n$. Order the point-pairs lexicographically. For the smallest point-pair $(a, b)$ not contained in a line, augment $G_{i}$ by adjoining a line $m$ containing only $a$ and $b$, and a line $m^{\prime}$ containing no point of $G_{i}$. If $k$ is a line of $G_{i}$ containing neither $a$ nor $b$, adjoint a new point $x_{k}$ on $k$ and $m$. If $l$ is any line of $G_{i}$, adjoin a new point $y_{1}$ on $l$ and $m^{\prime}$. Extend the ordering of the points of $G_{i}$ to the new points, and call the new structure $G_{i+1}$. Starting with $G_{0}$, the four point structure shown in Figure 3 defines $G_{\infty}$ to be the union of all the $G_{i}$ ( $i$ and integer).

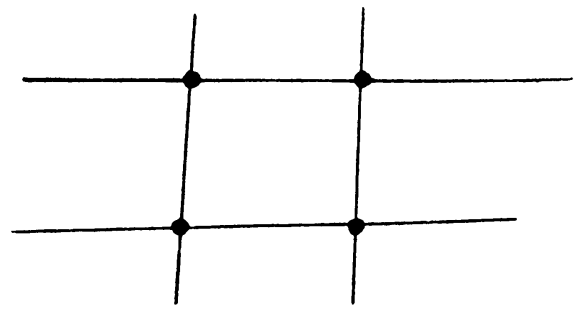

FIGURE 3

\section{REFERENCES}

[A1] E. Artin, Geometric algebra, Interscience, New York, 1957.

[B1] G. Birkhoff, Lattice theory (third ed.), Amer. Math. Soc. Colloq. Publ., vol. 25. Amer. Math. Soc.. Providence, R. I., 1967.

[B2] _ Combinatorial relations in projective geometry, Ann. of Math. (2) 36 (1935), 743-748.

[G1] S. Gorn, On incidence geometry, Bull. Amer. Math. Soc. 46 (1940), 158-167.

[H1] D. Hilbert, Foundations of geometry (transl. by E. J. Townsend), Open Court. LaSalle, Ill., 1902. (Reprinted, 1950)

[M1] F. Maeda and S. Maeda, Theon of symmetric lattices, Springer, New York, 1970.

[M2] K. Menger, New foundations of projective and affine geometry. Ann. of Math. (2) 37 (1936). 456-482.

[S1] U. Sasaki, Lattice theoretic characterization of geometries satisfying "Axiome der Verknüpfung". Hiroshima Math. J. Ser. A 16 (1953), 417-423.

[S2] Lattice theoretic characterization of affine geometry of arbitran dimension. Hiroshima Math. J. Ser. A 16 (1952), 223-238.

[S3] E. Snapper and R. Troyer, Metric affine geometrn, Academic Press, New York, 1971.

[W1] O. Wyler, Incidence geometn., Duke Math. J. 20 (1953), 601-610.

Department of Mathematics and Statistics, University of Massachusetts, Amherst, MasSACHUSETTS 01003 